\title{
Assessment of intestinal permeability of EGCG by piperine using Caco-2 cell monolayer system
}

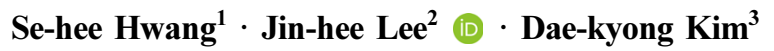

Received: 28 November 2019 / Accepted: 6 January 2020 / Published Online: 31 March 2020

(C) The Korean Society for Applied Biological Chemistry 2020

\begin{abstract}
EGGG), a flavonoid found in green tea, is known to have low bioavailability. In this study, we determine whether piperine, a natural bioenhancer, can increase the absorption rate of EGCG. Using a Caco-2 cell monolayer, permeability experiments were performed in Hanks' balanced salt solution (HBSS) and EGCG stability was adjusted to pH 6.5 and pH 5.5 by ascorbic acid treatment. When HBSS was adjusted to $\mathrm{pH} 6.5$, EGCG remained at $94.78 \%$ for up to $2 \mathrm{~h}$ and remained at $86.04 \%$ after $4 \mathrm{~h}$ and the net efflux decreased compared to the control. As a result, uptake was significantly increased in the piperine co-administered group compared to the EGCG-alone group, showing that piperine increased the permeability of EGCG in the Caco-2 cell monolayer. These results suggest that piperine inhibits EGCG glucuronidation and efflux, allowing for greater absorption of EGCG.
\end{abstract}

Keywords Ascorbic acid $\cdot$ Caco-2 cell $\cdot$ Efflux $\cdot$ Monolayer

Jin-hee Lee and Dae-kyong Kim are co-corresponding authors and contributed equally.

Jin-hee Lee $(\triangle)$

E-mail: jhlee@inewtree.com

Dae-kyong Kim $(\triangle)$

E-mail: proteinlab@daum.net

${ }^{1}$ College of Pharmacy, Chungang University, 84 Heukseok-ro, Dongjak-gu, Seoul 06974, Republic of Korea

${ }^{2}$ Health Food R\&D, Newtree Co., Ltd., 6-14, Baekjegobun-ro, 27-gil, Songpa-gu, Seoul 05604, Korea

${ }^{3}$ College of Pharmacy, Chungang University, 84 Heukseok-ro, Dongjak-gu, Seoul 06974, Republic of Korea

This is an Open Access article distributed under the terms of the Creative Commons Attribution Non-Commercial License (http://creativecommons. org/licenses/by-nc/3.0/) which permits unrestricted non-commercial use, distribution, and reproduction in any medium, provided the original work is properly cited.

\section{Introduction}

The use of natural products has a long history, and the development of natural supplements is ongoing due to their fewer side effects and easier access compared with pharmaceuticals. In vitro studies have shown significant results for natural products whereas the in vivo bioavailability and lipid solubility for these have not been always shown to be similar ones.

The major problem regarding bioavailability is the inability of these products to cross the lipid membranes of the intestine. The bioavailability can be improved with the use of novel delivery systems, such as liposomes, marinosomes, niosomes, and lipidbased systems that can enhance the rate of release as well as the capacity to cross lipid-rich biomembranes [1].

A "bioenhancer" is an agent capable of enhancing the bioavailability and bioefficacy of a particular drug, without any typical pharmacological activity of its own at the dose used [2].

The main component of green tea extract, catechin, contains the phenolic compound (-)-epigallocatechin gallate (EGCG) [3]. Compared to other beverages, green tea contains high levels of nitrogen, polyphenols, sugars, organic acids, vitamins, and minerals. Green tea polyphenols are mostly flavanols. The major green tea catechins are (+)-catechin (C), (-)-epicatechin (EC), (-)-epicatechin gallate (ECG), (-)-epigallocatechin (EGC), (-)-epigallocatechin gallate (EGCG), (-)-gallocatechin gallate $(\mathrm{GCG}),(+)$-gallocatechin (GC), and other alkaloids containing caffeine, theobromine, and theophylline [4].

The flavonoids extracted from green tea have very low bioavailability. One pharmacokinetic study showed that, after oral administration of green tea to rats, only $14 \%$ of EGC, $31 \%$ of EC, and $<1 \%$ of EGCG appeared in the blood [5]. For humans, after administration of $3 \mathrm{~g}$ of decaffeinated green tea, the maximum plasma concentrations for EGCG, EGC, and EC were 0.57, 1.60, and $0.6 \mathrm{M}$, respectively [6]. The apparent permeability coefficient (Papp) values of EGCG and EGCG niosomes were found to be $0.88 \pm 0.09$ and $1.42 \pm 0.24 \mathrm{~cm} / \mathrm{sec}$ in one in vitro study [3]. In another study, EGCG nanoformulations containing EGCG and 
piperine polymers in zein nanocarriers showed higher anticancer activity on various cell lines (HL60, SCC40, MCF7, HeLa, and Colo205) than pure EGCG [7]. The coadministration of piperine in CF-1 mice in another study improved the EGCG uptake in the small intestine by approximately 1.7-fold compared to the EGCGtreated group and increased by around 1.3-fold in plasma [8].

Piperine is a major component of the long pepper (Piper longum Linn) and the black pepper (Piper nigrum Linn). Both spices are commonly found in Asian diets [9]. Piperine can inhibit metabolism in the gut by inhibiting the enzyme CYP3A in epithelial cells, thereby enhancing bioavailability [10]. In addition, P-glycoprotein (P-gp) inhibition enhances bioavailability by inhibiting efflux during intestinal absorption [11]. For this reason, piperine may also enhance the intestinal absorption of EGCG.

Caco-2 cell monolayers are a useful experimental tool for evaluating the in vitro permeability of new compounds and may play an important role in the study of EGCG gastrointestinal permeability. In this study, we performed permeability experiments by creating experimental conditions to maintain the stability of EGCG in a Caco-2 cell monolayer. For EGCG stability evaluation, EGCG content was measured by HPLC in Hanks' balanced salt solution (HBSS) treated with bovine serum albumin (BSA), human serum albumin (HSA), hydrogen chloride, or ascorbic acid. We evaluated whether the permeability of EGCG is enhanced by piperine while maintaining a HBSS $\mathrm{pH}$ of 7.4-6.5, which is required for permeability evaluation in Caco- 2 cell monolayers.

\section{Materials and Methods}

\section{Materials}

In this experiment, EGCG ( $>98 \%$ ) was purchased from Tokyo Chemical Industry, Japan, piperine ( $>97 \%$ ) from was purchased from WAKO, and verapamil and ascorbic acid were purchased from Sigma. For cell culture, fetal bovine serum (FBS) and a $0.22-\mu \mathrm{m}$ bottle top filter system were purchased from Corning. Dulbecco's modified Eagle medium (DMEM), sodium biocarbonate solution, penicillin-streptomycin, and non-essential amino acid (NEAA) were purchased from Gibco. Cell proliferation kits (XTT, Welgene) were purchased for cytotoxicity experiments. Cell monolayer formation and transport experiments were performed using Corning ${ }^{\circledR} 24 \mathrm{~mm}$ Transwell-COL collagen-coated $0.4-\mu \mathrm{m}$ pore polytetrafluoroethylene (PTFE) membrane inserts and HBSS, Gibco). For HPLC analysis, acetonitrile (ACN) and methyl alcohol were purchased from Daejung at HPLC grade.

\section{Stability of EGCG on HBSS}

In order to measure the stability of EGCG depending on the $\mathrm{pH}$ condition, HBSS was adjusted to $\mathrm{pH} 3.5,4.5,5.5,6.5$, and 7.5 using $0.1 \mathrm{~N}$ hydrogen chloride. EGCG $(500 \mu \mathrm{M})$ was prepared in HBSS to make a $10 \%$ HBSS solution to the final $50 \mu \mathrm{M}$ EGCG while being incubated in a shaking incubator at $37^{\circ} \mathrm{C} \cdot 100-\mu \mathrm{L}$ aliquots of the above solution were taken at $0,1,2,3$, and $4 \mathrm{~h}$. In order to examine EGCG stability by BSA and HSA treatment, $1 \mathrm{mM}$ EGCG was prepared in HBSS and the concentrations of BSA and HSA used as stabilizers were prepared at $1.5 \mathrm{mM} .250$ $\mu \mathrm{L}$ of EGCG and stabilizer (BSA and HSA) were mixed to make a mixed reagent and was left to stand for 2 min. EGCG and PBS were used as the control. A 10\% HBSS solution was prepared using the reagents and then the experiment was conducted in the same manner as above. EGCG stability by ascorbic acid treatment was adjusted to $\mathrm{pH} 6.5$ and $\mathrm{pH} 5.5$ by adding $2.14 \mathrm{mM}$ and 3.75 $\mathrm{mM}$ of acid, respectively, to HBSS. 50 of $\mu \mathrm{M}$ EGCG was added to HBSS containing ascorbic acid and then reacted for $4 \mathrm{~h}$ by the same method as above, then the aliquots were analyzed by HPLC.

\section{Cell culture}

We purchased Caco-2 cells from Korea Cell Bank (Seoul, Korea) and added DMEM, 10\% FBS, 1\% NEAA and 1\% penicillinstreptomycin. We adjusted the solution to $\mathrm{pH} 7.0-7.3$ with $\mathrm{HCl}$ or $\mathrm{NaOH}$ and filtered it under reduced pressure using a $1 \mathrm{~L}$ bottle top filter system $(0.22 \mu \mathrm{m})$. For cell passage culture, we used medium containing NEAA. In the Caco-2 cell monolayer experiment, all compositions were the same, but we did not use NEAA. We placed cells in a $\mathrm{CO}_{2}$ incubator at $37{ }^{\circ} \mathrm{C}$ with $90 \%$ relative humidity and $5 \% \mathrm{CO}_{2}$. We replaced the culture medium 2-3 times per week. After growing up to $80-90 \%$ confluency, we detached the cells from the culture flask by adding a $0.25 \%$ trypsin-EDTA solution to seed in plates or flasks. In this experiment, we used cell passage numbers 19-28.

\section{Caco-2 cell monolayers}

Caco-2 cells were cultured as described above. We seeded Caco2 cells in 6-well plates with $0.4 \mu \mathrm{m}$ pore size collagen-coated inserts and PTFE membranes (Corning, USA) to form Caco- 2 cell monolayers. We placed $3 \mathrm{~mL}$ of DMEM medium on the basolateral side of the well, and dispensed $2 \mathrm{~mL}$ of cell solution containing $1 \times 10^{5}$ cells on the apical side. We incubated the 6 -well plate containing cells at $37{ }^{\circ} \mathrm{C}$ and $5 \% \mathrm{CO}_{2}$. After incubation for 48-72 h, we observed the cells by light microscopy, discarding the old media if the cells were attached, and replacing it with new media ( $3 \mathrm{~mL}$ on the basolateral side and $2 \mathrm{~mL}$ on the apical side). We performed media exchange of transwell cells every 3 days in the first week and every 2 days thereafter for a total of 21 days. Before carrying out the transport experiment, transepithelial electrical resistance measurements (Millicell ERS-2 Voltohmmeter) were taken to confirm the formation of Caco-2 cell monolayers. Before measurement, the probe was sterilized with $70 \%$ ethyl alcohol and soaked in DMEM. The 6-well plate to be measured in the incubator was brought to the clean bench, and the probe was placed on the apical and basolateral sides of the transwell. Only those with a value of $230 \Omega / \mathrm{cm}^{2}$ or more were used. If the measured epithelial electrical resistance was less than $230 \Omega / \mathrm{cm}^{2}$, tight junctions formed as differentiated Caco- 2 cells were not 
formed properly and these were excluded from transport experiments.

\section{Cytotoxity}

Caco-2 cells were diluted with DMEM to $1.510^{5}$ cells/well and $200 \mu \mathrm{L}$ of this dilution was then dispensed into 96 -well plates. The plates were incubated in a $5 \% \mathrm{CO}_{2}$ incubator at $37^{\circ} \mathrm{C}$ for $24 \mathrm{~h}$. For cytotoxic samples, the sample with the highest ratio of EGCG and piperine (1:2) and the sample mixed with EGCG and verapamil (1:0.5) were dispensed by concentration on a 96-well plate, which was then incubated for $4 \mathrm{~h}$ at $37^{\circ} \mathrm{C}$ in a $5 \% \mathrm{CO}_{2}$ incubator. After washing three times, $40 \mu \mathrm{L}$ of XTT solution was added and the absorbance was measured at 450 and $690 \mathrm{~nm}$ using an ELISA reader. The cell survival rate was obtained by selecting the cells which were treated with the test sample for $4 \mathrm{~h}$, mixing $20 \mu \mathrm{L}$ of this suspension with $20 \mu \mathrm{L}$ of trypan blue, and then dispensing on a counting slide to determine the cell count (NanoEnTek).

\section{Cellular transport studies}

The EGCG permeability and prediction of absorption in Caco-2 monolayers followed the procedure described previously [12] with some minor modifications. Six-well plates in which a Caco2 monolayer was incubated for 21 days were taken out of the incubator, the medium was removed, HBSS was used as a transport medium, and the plates were incubated for $1 \mathrm{~h}$. HBSS was used after adding $10 \mathrm{mM}$ ascorbic acid drop-by-drop and adjusting HBSS to $\mathrm{pH} 6.5$. After $1 \mathrm{~h}$, the HBSS was removed and $1.5 \mathrm{~mL}$ of each drug was added to the apical part of the plate to observe the experiment on the basolateral side from the apical side. After adding $2.5 \mathrm{~mL}$ of fresh HBSS to the basolateral side, $1 \mathrm{~mL}$ of the basolateral side was collected at $0.5,1,2,3$, and $4 \mathrm{~h}$ for analysis. To observe the transfer from the basolateral side to the apical side, the existing HBSS was removed, and $1.5 \mathrm{~mL}$ of HBSS without sample was added to the apical side of the plate and $2.5 \mathrm{~mL}$ of HBSS containing each sample was added to the basolateral side. During incubation at $37^{\circ} \mathrm{C}, 1 \mathrm{~mL}$ of sample was collected from the apical side at $0.5,1,2,3$, and $4 \mathrm{~h}$ and filtered through a syringe filter ( $13 \mathrm{~mm}, 0.45 \mu \mathrm{m}$, PVDF, Advantec) and then used for HPLC analysis. The Papp of EGCG was calculated using the following equation:

Papp $=d Q / d t \times \frac{1}{A \times C_{0}}$

where $d Q / d t$ is the permeability rate $(\mu \mathrm{g} / \mathrm{sec}), \mathrm{C}_{0}$ is the initial concentration in the donor chamber $(\mu \mathrm{g} / \mathrm{mL})$, and $\mathrm{A}$ is the surface area of the filter $\left(4.67 \mathrm{~cm}^{2}\right.$ in this study). After the transfer experiment, the 6-well plate with the Caco-2 monolayer was rinsed with HBSS, both apical and basal sides, $0.1 \mathrm{mg} / \mathrm{mL}$ lucifer yellow solution was added $(1.5 \mathrm{~mL})$ into the apical compartment, and HBSS buffer was added to the basal compartment. Plates were wrapped in foil and incubated for $1 \mathrm{~h}$ in a $37^{\circ} \mathrm{C}$ incubator while blocking light. Then $200 \mu \mathrm{L}$ was sampled from the basal compartment and measured using a $485 \mathrm{~nm}$ excitation filter and an emission filter of $527 \mathrm{~nm}$ on a fluorometer. The percentage of permeability was calculated from the fluorescence values as follows:

$\%$ permeability $=\frac{\text { samples }- \text { blank }}{\text { Luciferyellow }- \text { blank }} \times 100$

A permeability of $<3 \%$ is acceptable.

\section{Analysis by HPLC}

The analysis of EGCG using HPLC followed a method previously described with some minor modifications $[3,13,14]$. A Shiseido Nanospace SI-2 series HPLC system was used with two solvent pumps and a UV detector. For chromatography, a Shiseido Capcell Pak C 18 UG $120(5 \mu \mathrm{m}, 4.6 \mathrm{~mm} \times 250 \mathrm{~mm})$ was used and the column temperature was $40{ }^{\circ} \mathrm{C}$. The mobile phase used $0.1 \%$ acetic acid in distilled water and $0.1 \%$ acetic acid in acetonitrile. All samples were analyzed at a UV wavelength of $280 \mathrm{~nm}$, flow rate was $1 \mathrm{~mL} / \mathrm{min}$, injection volume was $35 \mu \mathrm{L}$, and the autosampler temperature was maintained at $4{ }^{\circ} \mathrm{C}$.

\section{Data analysis}

We performed all experiments at least three times. Data is expressed as the mean and the standard deviation $( \pm \mathrm{SD})$. We evaluated the significance of differences with Student's paired $t$ test in SPSS 21.0, setting the criterion of significance at $p<0.05$.

\section{Results and Discussion}

\section{EGCG stability on cellular assay}

In order to examine the stability of EGCG in vitro, the stability of EGCG on HBSS was measured by treatment with BSA and HSA, which are known to increase stability by protein binding. EGCG content was barely detected after $4 \mathrm{~h}$ in HBSS in the permeability assay. In contrast, the BSA-treated group showed increased EGCG stability. The BSA-treated group remained at around $57.19 \%$ EGCG after $2 \mathrm{~h}$. The HSA-treated group showed higher stability than the control group; however, EGCG stability was more effective in BSA than HSA (Fig. 1A). The level of protein binding was higher than $97 \%$ for both HSA and BSA.

The stability of EGCG in the weakly acidic and neutral range of HBSS buffer was surprisingly reduced. EGCG decreased to around $80 \%$ after $4 \mathrm{~h}$ in HBSS at $\mathrm{pH} 7.5$ and remained at around $100 \%$ after $4 \mathrm{~h}$ in HBSS at pH 3.5 (Fig. 1B). EGCG was found to be very unstable under $\mathrm{pH}$ neutral conditions. After $4 \mathrm{~h}$, EGCG stability under $\mathrm{pH} 5.5,4.5$, and 3.5 conditions remained at 80 , 100 , and $100 \%$, respectively, but permeability experiments were difficult in vitro due to low $\mathrm{pH}$ values.

In consideration of the decrease of stability by redox reaction in neutral conditions, EGCG was treated with ascorbic acid to 
(A)

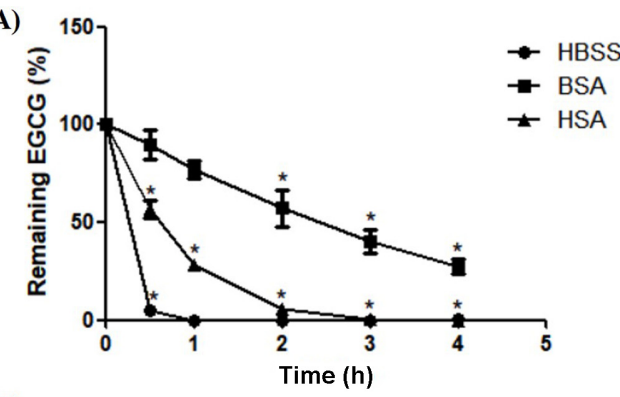

(B)

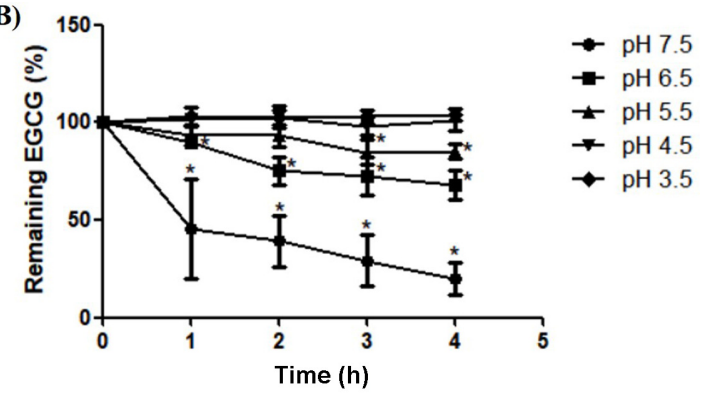

(C)

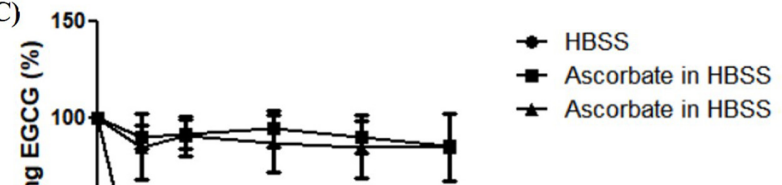

Fig. 1 Stability profiles of $50 \mu \mathrm{M}$ of EGCG in HBSS buffer $\left(37^{\circ} \mathrm{C}\right)(\mathrm{A})$ in the presence of BSA or HSA (B) after adjusting $\mathrm{pH}$ condition using $\mathrm{HCl}$ and $(\mathrm{C})$ in the presence of ascorbic acid. Data points represent mean \pm $\mathrm{SD}(\mathrm{n}=3)$. *Significantly different from zero time, $p<0.05, * *$ significantly different from zero time, $p<0.01$

evaluate its stability in weak alkaline conditions. In HBSS adjusted to $\mathrm{pH} 6.5$ by ascorbic acid, EGCG remained at $94.78 \%$ for up to $2 \mathrm{~h}$, and remained at $86.04 \%$ after $4 \mathrm{~h}$. There was no significant difference between $\mathrm{pH} 6.5$ and $\mathrm{pH} 5.5$. In vitro permeability of HBSS at pH 6.5 with ascorbic acid is expected to yield at least $85 \%$ of EGCG after $4 \mathrm{~h}$ of sampling (Fig. 1c).

In many studies, the stability of EGCG has been tested using cysteine, GSH, BSA, has, and ascorbic acid [14-17]. In one study, the stability of EGCG in the presence of three thiol-containing species [cysteine (Cys), glutathione (GSH) and 3-mercaptohexan1-ol (3SH)] was followed under both neutral and acidic conditions. Both Cys and GSH increased the rate of EGCG oxidation at pH 4. At $\mathrm{pH} \mathrm{7,} \mathrm{only} \mathrm{Cys} \mathrm{was} \mathrm{found} \mathrm{to} \mathrm{increase} \mathrm{the} \mathrm{rate} \mathrm{of} \mathrm{EGCG}$ oxidation. In this experiment, cysteine was not suitable for the purpose of increasing the stability of EGCG under the conditions that can be tested in the cell [15]. Serum albumin is known to maintain stability through complex with EGCG. Using this property, coated BSA epigallocatechin gallate nanoparticles were prepared and high stability and permeability were confirmed in human intestinal epithelial Caco-2 cells [14]. In another study, it was reported that EGCG can form large water-soluble complexes with BSA or HSA and the complexes are stable to denaturation by detergents [18]. In this study, both BSA and HSA increased the stability of EGCG, and BSA showed higher stability than HSA. However, although viability could be improved in cell experiments, the stability of EGCG was not sufficient. Another study examined the effect of ascorbic acid and citric acid on the stability of green tea catechins (GTCs) as a mixture of EC, ECG, EGC, and EGCG, incubated in sodium phosphate buffer ( $\mathrm{pH}$ 7.42). Ascorbic acid added to the incubation mixture significantly increased the stability of GTCs, whereas citric acid exhibited no effect. Four epicatechin derivatives examined demonstrated varying stability, with EGCG and EGC being equally instable and EC and ECG being relatively stable. The addition of ascorbic acid significantly increased the stability of all four derivatives, particularly EGC and EGCG [17].

In our experiment, the $\mathrm{pH}$-controlled experiments showed that the stability of EGCG was increased only when the $\mathrm{pH}$ was changed to 4.5-3.5. In the study of Song et al. [3], the stability of EGCG in niosomes of EGCG was also tested in HBSS buffer $\mathrm{pH}$ 7.4 using ascorbic acid $(10 \mathrm{mM})$. The final $\mathrm{pH}$ is expected to be acidic; so, in our experiment, the smallest amount of ascorbic acid was tested to find the section that could be tested. As a result, when HBSS was treated to $\mathrm{pH} 6.5$ using ascorbic acid, it was selected as a method to stabilize EGCG while enabling cell experiments. According to one study with a decrease in $\mathrm{pH}$ from 7.4 to 5.0, there was a 2.2-fold increase in the cellular accumulation of ECG and GTC [19].

In cell uptake experiments, changes in $\mathrm{pH}$ can affect the transport of material, so experiments at least in the range are important. In experiments that determinate drug absorption and drug permeability through a Caco- 2 monolayer, the $\mathrm{pH}$ of HBSS is recommended to be 7.4 but, depending on the type of experiment, the acid microclimate of the small intestine can be mimicked and the $\mathrm{pH}$ of the apical compartment can be adjusted to $\mathrm{pH} 6.5$ [12].

\section{Cytotoxicity}

In the permeability experiment, EGCG and piperine were mixed to be co-administered at high concentration $(1: 2)$, and the cytotoxicity in Caco-2 cells was measured. To measure their cytotoxic effects, EGCG and piperine were mixed up to 250 and $500 \mu \mathrm{M}$, respectively, diluted in multiples of two, and administered at a minimum of 3.90625 and $7.8125 \mu \mathrm{M}$. The cell viability of the highest concentration of EGCG and piperine was $7 \%(p<0.001)$. 


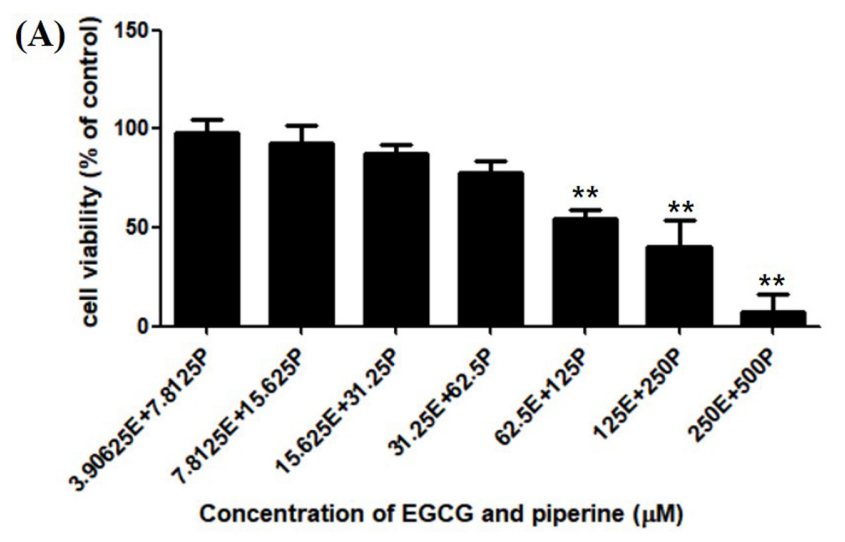

(B)

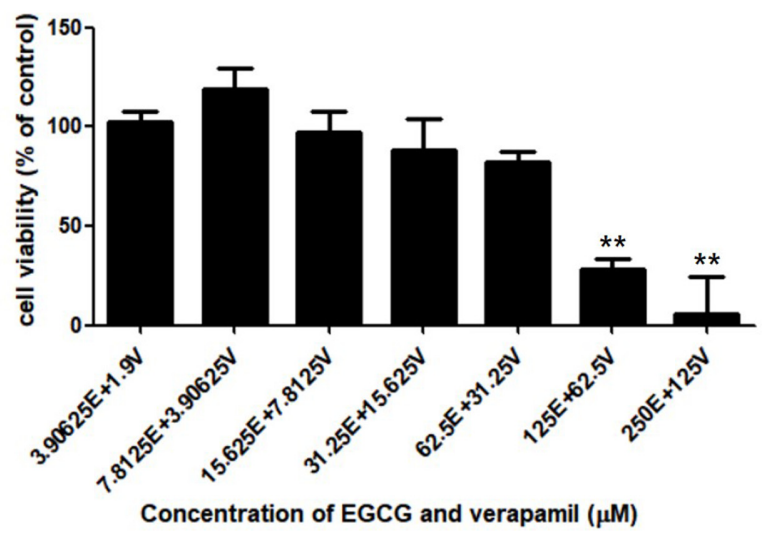

Fig. 2 Viability of Caco-2 cells exposed to (A) EGCG and piperine (1:2), (B) EGCG and verapamil (1:0.5) for $4 \mathrm{~h}$. Data points represent mean \pm SD $(\mathrm{n}=3)$. *Significantly different from control group, $p<0.05$, $* *$ significantly different from control group, $p<0.01$

The viability of the EGCG with piperine (7.8125 and $15.625 \mu \mathrm{M}$, respectively) group was $92.95 \%$ with no significant change compared to the control. At EGCG and piperine concentrations of 31.25 and $62.5 \mu \mathrm{M}$, cell viability was decreased to $77.41 \%$ (Fig. $2 \mathrm{~A}$ ). When verapamil was used as a positive control, the cell viability of EGCG $31.25 \mu \mathrm{M}$ and verapamil $15.625 \mu \mathrm{M}$ was $88.57 \%$, with no significant change (Fig. 2B).

EGCG is a strong antioxidant and is characterized by its color reaction with XTT reagent. While going through the process of washing several times, it is important that cells are conserved, as they can easily fall from the plate. The treatment capacity was determined at a viability level of about $80 \%$ and the survival rate compared to the control was measured by cell counting for each dose. The survival rate for the EGCG and piperine doses used in the permeability test was $97 \pm 3 \%$. The survival rate in the group receiving EGCG and verapamil was $81 \pm 8 \%$ (Table 1 ). The dose used in the XTT cytotoxicity experiment showed no cytotoxic effect on Caco-2 cells.

According to previous research, EC, EGCG, and GA (gallic acid) were found to be non-toxic at concentrations ranging between 100 and $250 \mu \mathrm{M}$ with a cellular survival rate of $70-80 \%$, whereas a $60 \%$ cytotoxicity occurred at a concentration of
Table 1 Survival percent of Caco-2 cells after 24-h treatment with EGCG and piperine

\begin{tabular}{lc}
\hline \hline \multicolumn{1}{c}{ Treatment } & Survival rate (\%) \\
\hline Control & - \\
EGCG $(31.25 \mu \mathrm{M})$ & $96 \pm 23$ \\
EGCG and piperine $(31.25$ and $62.5 \mu \mathrm{M})$ & $97 \pm 3$ \\
EGCG and verapamil $(31.25$ and $15.625 \mu \mathrm{M})$ & $81 \pm 8$ \\
\hline
\end{tabular}

$500 \mu \mathrm{M}$ for all flavonoids tested in Caco-2 cells [20]. In this experiment, we tested the toxicity of EGCG on Caco-2 cells with a sample of piperine $(500 \mu \mathrm{M})$ and verapamil $(125 \mu \mathrm{M})$ by applying the highest ratio of piperine and verapamil to be administered together with $250 \mu \mathrm{M}$ of EGCG based on these concentrations. When cell viability is lowered due to toxicity, the tight junction of the monolayer can be released. After the experiment, the tight junction was reconfirmed by checking the level of permeability with lucifer yellow.

EGCG accumulation on Caco-2 cell monolayer with piperine The EGCG uptake content, according to the piperine content, was tested in the Caco- 2 cell monolayer. The level of EGCG uptake increased with time, and an increase in uptake showed in the piperine-treated group $(0.5,1.0$, and 2.0 times of EGCG) compared to the control group. In particular, the uptake in the experimental group treated with piperine (2.0 times of EGCG) was confirmed to increase significantly after $1 \mathrm{~h}(p<0.05)$. This result was similar to the verapamil treatment group (Fig. 3a).

The transport of phenolic compounds to the cell is affected by a certain type of protein transporter, and hydrophilic compounds can be transported to the enterocytes by SGLT1. Glucuronides and sulfates of polyphenolic compounds in the small intestine are too hydrophilic to enter the cell by simple diffusion [21]. In this process, ATP-binding cassettes act, including multidrug resistance proteins (MRPs), breast cancer resistance proteins (BCRP, ABCG2), and P-gp. MRP2 is responsible for transporting polyphenols, such as EGCG, with glucuronide and sulfate to the membranes of enterocytes [22]. P-gp is also responsible for efflux transport.

Transepithelial transport of EGCG in Caco-2 cells with piperine Little is known about absorptive and efflux transporters in relation to transepithelial transport of EGCG. However, glucuronides or sulfates make the material very hydrophilic for simple diffusion. Absorbed material are known to be effluxed by MRPs and Pglycoproteins. In this study, we also performed transepithelial transport assays to determine the effect of piperine on this mechanism. Simultaneous administration of piperine increased the Papp for basal to apical and apical to basal transport of EGCG. In the experimental group treated with piperine $(0.5,1.0$, and 2.0 times of EGCG), Papp A to B tended to increase to $3.741 \pm 2.14$, $5.784 \pm 3.08$, and $5.864 \pm 3.05 \times 10^{6} \mathrm{~cm} / \mathrm{sec}$, respectively. In addition, Papp B to A showed a tendency to increase to $1.731 \pm 1.57$, 


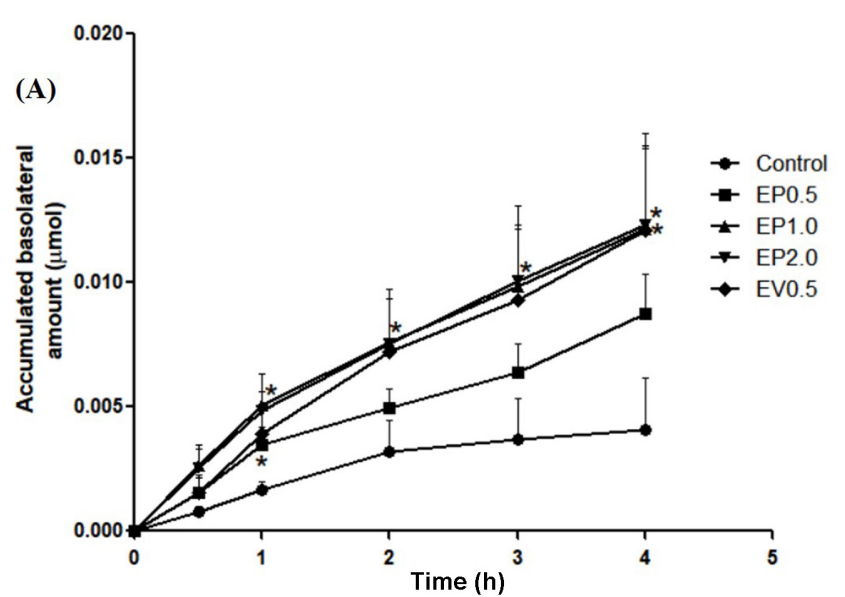

(B)

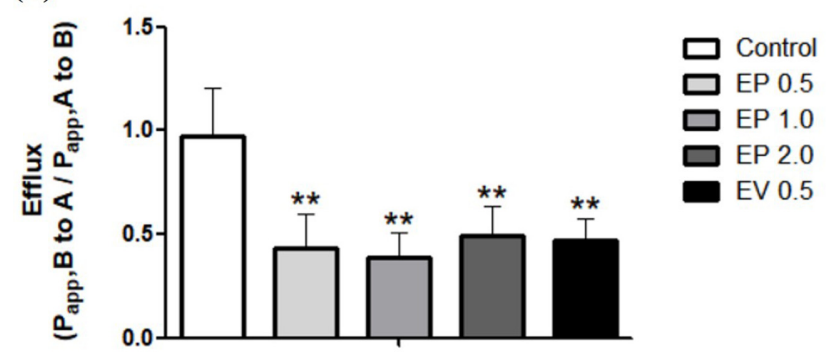

(C)

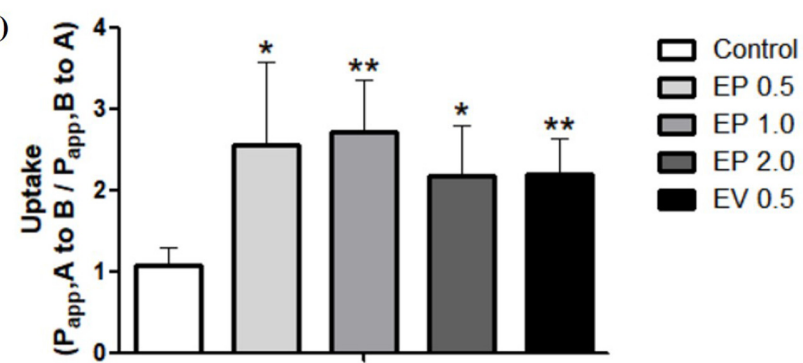

Fig. 3 Permeability of EGCG with piperine on Caco-2 cell monolayers (A) Accumulated amount of EGCG in the basolateral chamber during the 4-h experiment. (B) Uptake is calculated as the ratio of apical-to-basal apparent permeability coefficient (Papp) to basal-to-apical Papp for $4 \mathrm{~h}$. (C) Efflux is calculated as the ratio of basal-to-apical Papp to apical-tobasal Papp for $4 \mathrm{~h}$. Data points represent mean \pm SD $(n=3)$ *Significantly different from control group, $p<0.05$, **significantly different from control group, $p<0.01$. EP0.5, EGCG $31.25 \mu \mathrm{M}$ and piperine $15.625 \mu \mathrm{M}$; EP1.0, EGCG $31.25 \mu \mathrm{M}$ and piperine $31.25 \mu \mathrm{M}$ EP2.0, EGCG $31.25 \mu \mathrm{M}$ and piperine $62.5 \mu \mathrm{M}$; EV0.5, EGCG $31.25 \mu \mathrm{M}$ and verapamil $15.625 \mathrm{iM}$

$2.114 \pm 0.988$, and $2.772 \pm 1.32 \times 10^{6} \mathrm{~cm} / \mathrm{sec}$, respectively (Table 2). However, the increase to A to B was larger than the increase to B to A. Due to this effect, the net efflux decreased compared to the control. As a result, uptake (Papp A to B/Papp B to A) was significantly increased in the piperine co-administered group compared to the EGCG-alone group, and significant decreases were found in the efflux (Papp B to A/Papp A to B) in the 0.5-, $1.0-$, and 2.0-fold treatment groups compared to EGCG $(p<0.01)$. The same phenomenon was observed in the glucuronidase
Table 2 The apparent permeability coefficients (Papp) and efflux (Papp B to A/Papp A to B) of EGCG treated with piperine

\begin{tabular}{lccc}
\hline \hline \multirow{2}{*}{\multicolumn{1}{c}{ Treatment }} & \multicolumn{2}{c}{ Papp $\left(\times 10^{-6} \mathrm{~cm} / \mathrm{s}\right)$} & Efflux \\
\cline { 2 - 4 } & A to B & B to A & \\
\hline Control & $1.907 \pm 1.99$ & $1.700 \pm 1.85$ & 0.970 \\
Piperine $0.5(15.625 \mu \mathrm{M})$ & $3.741 \pm 2.14$ & $1.731 \pm 1.57$ & 0.435 \\
Piperine $1.0(31.25 \mu \mathrm{M})$ & $5.784 \pm 3.08$ & $2.114 \pm 0.88$ & 0.389 \\
Piperine $2.0(62.5 \mu \mathrm{M})$ & $5.864 \pm 3.05$ & $2.772 \pm 1.32$ & 0.494 \\
Verapamil $0.5(15.625 \mu \mathrm{M})$ & $5.349 \pm 4.19$ & $2.234 \pm 1.55$ & 0.472 \\
\hline
\end{tabular}

inhibitor and the non-selective first generation P-gp inhibitor verapamil group (Figs. 3B, C).

In this experiment, we evaluated the permeability of EGCG on a Caco-2 monolayer by treating it with the bioenhancer piperine, which is known to have glucuronidase and P-gp inhibitory effects. As a result, it was confirmed that the Papp from apical to basal increased the piperine level in concentration-dependent manner. This showed a clear concentration dependence compared to the Papp from basal to apical, suggesting that the glucuronidation of EGCG was inhibited and the absorption increased without a change to the hydrophilic form. These finding correlate with those of other studies where piperine improved the bioavailability of (-)-epigallocatechin-3-gallate in mice. Glucuronidation of EGCG in the hepatic and small intestinal microsomes of mice was measured by piperine concentration. In small intestinal microsomes, glucuronidation decreased significantly with piperine concentration, at 100 and $500 \mu \mathrm{mol} / \mathrm{L}$, piperine inhibited small intestinal glucuronidation of EGCG by $40 \%$ and $60 \%$, respectively [8].

In addition, piperine is known as an inhibitor of P-gp, which is expected to have an efflux inhibitory effect on EGCG. It is reported that the efflux mechanism of EGCG contains not only Pgp but also MRP2 [23,24]. Further investigation is required to determine how efflux of EGCG can be inhibited. Verapamil was also observed in the effects of EGCG on the pharmacokinetics of verapamil in rats. When the 0,2 , and $10 \mathrm{mg} / \mathrm{kg}$ of EGCG were administered, the AUC of verapamil was increased to $280 \pm 80.0$, $488 \pm 112$, and $591 \pm 137$, respectively. The presence of EGCG, rather than the difference in dose, seems to promote the absorption of verapamil, which was observed by CYP3A and P-gp inhibition of EGCG [25].

Piperine increased the permeability of EGCG in our Caco- 2 cell monolayer. This suggests that piperine has the effect of inhibiting EGCG glucuronidation and efflux. Ascorbic acid treatment was an effective method for the permeability assay of EGCG in vitro. It is expected that in vitro methods may be more conveniently used in developing functional foods or drugs using EGCG in the future; however, more in-depth mechanistic studies are needed to determine the exact uptake and efflux mechanisms. These findings of this study suggest that the addition of piperine to EGCG containing food or drug should improve the bioavailability of EGCG, demonstrating the potential for more effective functions in 
vivo, enhancing the practical efficacy with low doses. The EGCG would actually remain in the forms of EGCG or further metabolites by metabolic enzymes in human body, where the bioavailable forms of the compounds may be elucidated by further studies in vivo and in human clinical trials.

Acknowledgment This research was supported by ICT and Future Planning through the National Research Foundation (Bio-Synergy Research Project NRF 2013M3A9C4078159) of the Ministry of Science.

\section{References}

1. Uchegbu IF, Vyas SP (1998) Non-ionic surfactant based vesicles (niosomes) in drug delivery. Int J Pharma 172(1-2): 33-70

2. Kesarwani K, Gupta R, Mukerjee A (2013) Bioavailability enhancers of herbal origin: An overview. Asian Pac J Trop Biomed 3(4): 253-266

3. Song Q, Li D, Zhou Y, Yang J, Yang W, Zhou G, Wen J (2014) Enhanced uptake and transport of (+)-catechin and (-)-epigallocatechin gallate in niosomal formulation by human intestinal Caco-2 cells. Int $\mathrm{J}$ Nanomedicine 9: 2157-2165

4. Graham HN (1992) Green tea composition, consumption, and polyphenol chemistry. Prev Med 21: 334-350

5. Chen L, Lee MJ, Li HE, Yang CS (1997) Absorption, distribution, and elimination of tea polyphenols in rats. Drug Metab Dispos 25: 1045 1050

6. Yang CS, Chen L, Lee MJ, Balentine D, Kuo MC, Schantz SP (1998) Blood and urine levels of tea catechins after ingestion of different amounts of green tea by human volunteers. Cancer Epidemiol Biomark Prev 7(4): 351-354

7. Dahiya S, Rani R, Dhingra D, Kumar S, Dilbaghi N (2018) Conjugation of epigallocatechin gallate and piperine into a zein nanocarrier: implication on antioxidant and anticancer potential. Adv Nat Sci Nanosci Nanotechnol 9: 035011

8. Lambert JD, Hong J, Kim DH, Mishin VM, Yang CS (2004) Piperine enhances the bioavailability of the tea polyphenol (-)-epigallocatechin-3gallate in mice. J Nutr 134(8): 1948-1952

9. Han Y, Chin Tan TM, Lim LY (2008) In vitro and in vivo evaluation of the effects of piperine on P-gp function and expression. ýToxicol Appl Pharmacol 230: 283-289

10. Wadhwa S, Singhal S, Rawat S (2014) Bioavailability Enhancement by piperine: A review. Asian J Biomed Pharmaceut Sci 04(36): 1-8

11. Jin MJ, Han HK (2010) Effect of piperine, a major component of black pepper, on the Intestinal Absorption of fexofenadine and Its Implication on food-drug interaction. J Food Sci 75(3): 93-96
12. Hubatsch I, Ragnarsson EG, Artursson P (2007) Determination of drug permeability and prediction of drug absorption in Caco-2 monolayers. Nature protocols 2(9): 2111-2119

13. Zhang L, Zheng Y, Chow MS, Zuo Z (2004) Investigation of intestinal absorption and disposition of green tea catechins by Caco-2 monolayer model. Int J pharm 287(1-2): 1-12

14. Li Z, Ha J, Zou T, Gu L (2014) Fabrication of coated bovin serum albumin(BSA)-epigallocatechin gallate (EGCG) nanoparticles and their transport across monolayers of human intestinal epithelial Caco-2 cells. Food Funct 5: 1278-1285

15. Unnadkat NR, Elias RJ (2012) Oxidative stability of (-)-epigallocatechin gallate in the presence of thiols. J Agric Food Chem 60(43): 1081510821

16. Zhang Y, Xu YY, Sun WJ, Zhang MH, Zheng YF, Shen HM, Yang J, Zhu XQ (2016) FBS or BSA Inhibits EGCG Induced Cell Death through Covalent Binding and the Reduction of Intracellular ROS Production. Biomed Res Int 2016: 5013409

17. Chen ZY, Zhu QY, Wong YF, Zhang Z, Chung HY (1998) Stabilizing Effect of Ascorbic Acid on Green Tea Catechins. J Agric Food Chem 46(7): 2512-2516

18. Nozaki A, Hori M, Kimura T, Lto H, Hatano T (2009) Interaction of polyphenols with proteins: binding of (-)-epigallocatechin gallate to serum albumin, estimated by induced circular dichroism. Chem Pharm Bull 57(2): 224-228

19. Vaidyanathan JB, Walle T (2003) Cellular uptake and efflux of the tea flavonoid (-)-epicatechin-3-gallate in the human intestinal cell line caco2. J Pharmacol Exp Ther 307(2): 745-752

20. Salucci M, Stivala LA, Maiani G, Bugianesi R, Vannini V (2002) Flavonoids uptake and their effect on cell cycle of human colon adenocarcinoma cells (Caco2). Br J Cancer 86(10): 1645-1651

21. Lewandowska U, Szewczyk K, Hrabec E, Janecka A, Gorlach S (2013) Overwiew of metabolism and bioavailability enhancement of polyphenols. J Agric Food Chem 61: 12183-12199

22. Alvarez AI, Real R, Perez M, Mendoza G, Prieto JG, Merino G (2010) Modulation of the activity of ABC transporters (P-glycoprotein, MRP2, BCRP) by flavonoids and drug response. J Pharm Sci 99: 598-617

23. Jodoin J, Demeule M, Beliveau R (2002) Inhibition of the multidrug resistance P-glycoprotein activity by green tea polyphenols. Biochimica et Biophysica Acta 1542: 149-159

24. Hong J, Lambert JD, Lee SH, Sinko PJ, Yang CS (2003) Involvement of multidrug resistance-associated proteins in regulating cellular levels of (-)-epigallocatechin-3-gallate and its methyl metabolites. Biochem Biophys Res Commun 310(1): 222-227

25. Chung JH, Choi DH, Choi JS (2009) Effects of oral epigallocatechin gallate on the oral pharmacokinetics of verapamil in rats. Biopharm Drug Dispos 30: 90-93 